\title{
Ørsted and Magsat scalar anomaly fields
}

\author{
D. J. Ivers ${ }^{1}$, R. J. Stening ${ }^{2}$, J. Turner ${ }^{1}$, and D. E. Winch ${ }^{1}$ \\ ${ }^{1}$ School of Mathematics and Statistics, University of Sydney, Sydney 2006, Australia \\ ${ }^{2}$ School of Physics, University of New South Wales, Sydney 2052, Australia
}

(Received April 12, 2000; Revised August 9, 2000; Accepted August 9, 2000)

\begin{abstract}
The scalar anomaly field determined from available Ørsted data is compared with the upward continued scalar anomaly field derived from Magsat data. Two techniques were used to remove the core field from the Ørsted satellite data. In the first method, monthly spherical harmonic core field models of degree and order 13 derived from scalar and vector data were subtracted, and in the second method, along-track high-pass filtering of scalar data only was used. In both methods, the binned residuals were interpolated to a sphere, and subsequently filtered. Monthly degree and order 13 spherical harmonic core field models were removed from Magsat vector data. The binned Magsat vector residuals were interpolated to a sphere, filtered, and upward continued by high degree spherical harmonic analysis. The corresponding Magsat scalar anomaly field at Ørsted altitude was then determined. For latitudes below 50 degrees, removal of the core field by signal processing techniques from presently available Ørsted data led to a scalar anomaly field in better agreement with that determined from Magsat data, than removal by spherical harmonic analysis.
\end{abstract}

\section{Introduction}

Langel and Hinze (1998) give an encyclopaedic summary of the state of the analysis of satellite magnetic data, and refer to the understanding of the Earth's lithosphere gained by gathering and analyzing magnetic data. The recent availability of Ørsted data following its launch in 1999 has given a new impetus to these studies.

Separation of the scalar anomaly field from satellite data requires an explicit or implicit removal of a core field model derived from that data. The explicit core field model consists of spherical harmonic coefficients to degree and order 13 in a scalar potential. An implicit core field model in Ørsted data was removed by signal processing techniques, involving high-pass filtering of the satellite data along each track. (See Section 5.)

Ørsted monthly core field models were estimated by a series of spherical harmonic analyses of scalar and vector data. Data with large residuals were removed until finally all remaining data had residuals of less than $50 \mathrm{nT}$. (See Sections 3 and 4). The monthly core field models for Magsat data were estimated by spherical harmonic analysis of vector data with residuals of less than $50 \mathrm{nT}$. (See Section 7.)

For each satellite and for each scalar anomaly field extraction technique, the residual values over all months were collected into 1-degree by 1-degree bins. The calculation of the scalar residuals requires separate determination of both the scalar model field and the scalar total field (model + residual) from the respective vector components. The scalar residual field is then the difference between these two. In the case of the along track filtering method, the scalar residuals are of

Copy right(C) The Society of Geomagnetism and Earth, Planetary and Space Sciences (SGEPSS); The Seismological Society of Japan; The Volcanological Society of Japan; The Geodetic Society of Japan; The Japanese Society for Planetary Sciences. course produced immediately.

Simply averaged within each bin, the contours of vector field residual values showed furrows corresponding to sections of satellite orbits. When the vector and scalar field residual values were linearly interpolated to the centre of each bin and to a sphere of radius, $7130 \mathrm{~km}$ for Ørsted and $6800 \mathrm{~km}$ for Magsat, the satellite track furrows in the contours were greatly reduced but still apparent. (See Section 6.) These effects were successfully minimized with the application of a spatial notch filter imposed just prior to plotting. Additional high and low pass spatial filtering was incorporated into the notch filter to eradicate unwanted features.

In order to compare more accurately Magsat and Ørsted residuals, a degree 14 and higher spherical harmonic potential expansion was obtained from the binned, cell centered and altitude compensated Magsat vector residuals before any other processing was initiated. (See Section 8.) This high degree spherical harmonic expansion is separate from the expansion of degree and order 13 and less obtained for the monthly core models. The Magsat $X, Y$ and $Z$ components for an average model field and the corresponding total field $F$ were then synthesized separately from the potential expansions at the mean Ørsted radius. Thereafter the equivalent core scalar and main scalar fields were derived and, from the difference of these, the Magsat anomaly field seen at mean Ørsted radius was obtained.

For latitudes below 50 degrees, the extrapolated Magsat total intensity scalar anomaly field over continental regions correlated better with the Ørsted scalar anomaly field obtained by high-pass along track filtering than with that obtained using a core model field based on spherical harmonic analysis. (See Section 9.)

A flow chart for the calculations is given as Fig. 14. 


\section{The Ørsted Satellite and Instrumentation}

An initiative of the Danish Meteorological Office, the Ørsted satellite was launched from Vandenberg on 23 February 1999 at 10:29:55 UT. The altitude varies between 649 $\mathrm{km}$ at perigee and $865 \mathrm{~km}$ at apogee, which places it somewhat above the Magsat satellite launched in November 1979 , whose altitude ranged from 325 to $550 \mathrm{~km}$. Ørsted was launched in a polar orbit with an inclination of $96.48 \mathrm{deg}$, and placed in an almost Sun-synchronous orbit with the longitude of the ascending node at 14:11 local time, with a nodal drift moving the ascending node towards noon.

The Ørsted satellite carries two magnetometers, a Compact Spherical Coil (CSC) flux-gate magnetometer to measure the Earth's magnetic vector field, and an Overhauser (OVH) magnetometer to measure the total intensity of the Earth's main magnetic field. Primary attitude determination of the satellite is by a star-imager camera.

The Ørsted CSC vector magnetometer needs to be calibrated at regular intervals, like all fluxgate magnetometers. Olsen et al. (2000) describe the mathematical details of the processes of in-flight CSC calibration. Applying a known magnetic vector field to the instrument gives vector calibration, and using the total intensity from the $\mathrm{OVH}$ instrument gives scalar calibration.

\section{3. Ørsted Data and Data Selection}

The Ørsted satellite provides two data sets-scalar data from the Overhauser (OVH) scalar magnetometer and high precision magnetic field component (MAG-L) data from the Compact Spherical Coil (CSC) fluxgate magnetometer. Scalar and vector data samples are given at one-second intervals. A total of 109 days of MAG-L data, and a total of 181 days of $\mathrm{OVH}$ data being about $336 \mathrm{Mb}$ and $652 \mathrm{Mb}$ respectively, have been used. Figure 1 shows the distributions of days for which the OVH and MAG-L data are available.

There were 5,682,207 Ørsted scalar data of which $2,834,101$ were accepted. There was a smaller amount of Ørsted vector data, there being 2,651,406 vector data, of which 1,203,401 were accepted. Magsat provided vector data only, there being 29,178,046 vector data, of which $6,311,914$ were accepted.

Figure 2 shows the number of measurements, or 'hits' in each 1 degree by 1 degree bin for nighttime Ørsted vector data. Where there are no 'hits' the bin is white. The limited amount of data over the South Atlantic anomaly is due to incorrect parameter settings of the star imager camera during the first months of the mission. Figure 3 shows the corresponding distribution for Magsat dawn data.

There is a distribution of discrepancies of a few nanoTeslas between the scalar total intensity recorded on the $\mathrm{OVH}$ magnetometer and that computed from the vector field components measured on the MAG-L instrument. Extremum differences of $-44.0 \mathrm{nT}$ and $16.9 \mathrm{nT}$ were found, with an average value of $-0.2 \mathrm{nT}$ and an rms value of $1.0 \mathrm{nT}$. See Fig. 4.

Olsen (2000) has also recently reported strange signatures in Ørsted vector data. Ørsted observations minus synthetic values from a field model had a rather large scatter of values up to a few tens of nanoTeslas, with a signature almost entirely in the direction $\mathbf{n} \times \mathbf{B}$, where $\mathbf{n}$ is the direction of the

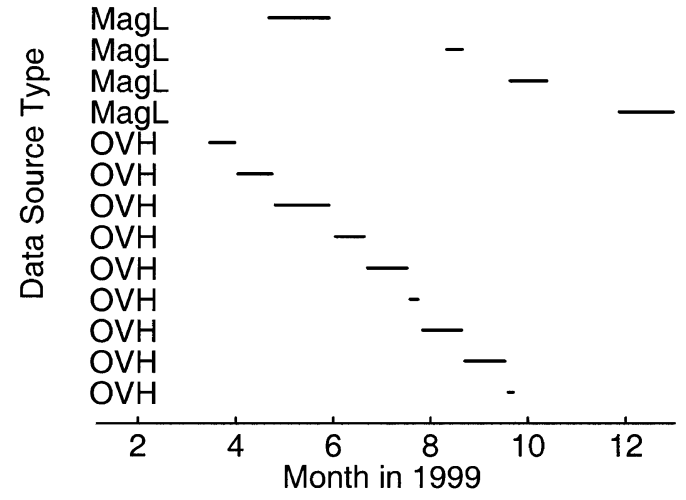

Fig. 1. The availability of Ørsted vector (MAG-L) data and scalar (OVH) data.

bore-sight of the Ørsted star imager.

More consistent crustal fields were determined from the available MAG-L vector data than from OVH scalar data. For this reason, although the scalar and vector data have been used together to determine the Ørsted core model, only vector data were then used to determine the crustal field. The OVH instrument is located halfway along the boom, and the CSC instrument is at the end. Stray fields from the satellite may have had a greater influence on the $\mathrm{OVH}$ instrument than on the CSC instrument.

No planetary magnetic three-hour-range index ( $K p$ index) was included with the OVH data. Where the $K p$ index was included, MAG-L data was chosen with $K p<2$.

\section{4. Ørsted Core and Anomaly Field Determination}

Spherical harmonic analysis to degree and order 13 was used to determine the core field and to reject data from all further analysis. Outlier observations or records were rejected at each step of a ten-step iterative spherical harmonic analysis of combined scalar $(\mathrm{OVH})$ and vector $(\mathrm{MAG}-\mathrm{L})$ data for nighttime sections of orbits, until only those observations or records with a residual of less than $50 \mathrm{nT}$ remained. When the MAG-L data included an OVH scalar value, the total intensity determined from the MAG-L data was required to be within $10 \mathrm{nT}$ of the $\mathrm{OVH}$ value.

Monthly models of the core field to degree and order 13 were determined by this iterative spherical harmonic analysis of the selected Ørsted MAG-L vector and OVH scalar night data for April, June, September, and December. The models included three first order external field terms. The core field terms were linearly interpolated for missing months of vector data. From the core field and the filtered vector residual known in each 1 degree by 1 degree bin, a scalar field was determined for the core field plus vector residual field and for the core field alone. The difference between these scalar fields is then the scalar anomaly field. It is assumed that, at least over the satellite's lifetime, there is no secular variation in the crustal field, and therefore there is no secular variation in the scalar anomaly field determined for different months.

After the models were determined, they were then used to extract the scalar anomaly field values, which were collected in 1 degree by 1 degree bins. A simple average of the scalar anomaly field values in each bin gave rise to furrows in oth- 

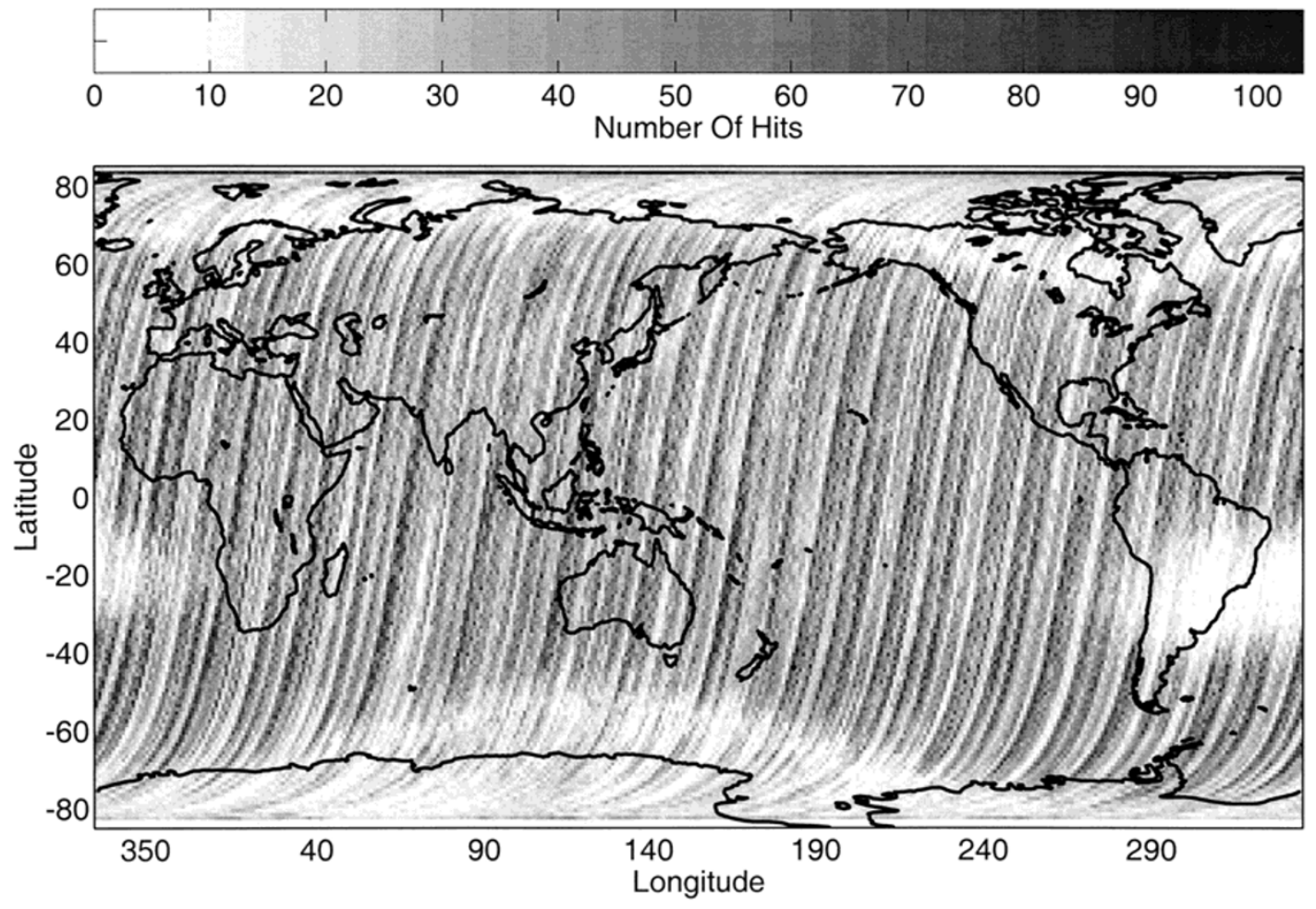

Fig. 2. Number of hits in each 1-degree by 1-degree bin, Ørsted nighttime vector data.
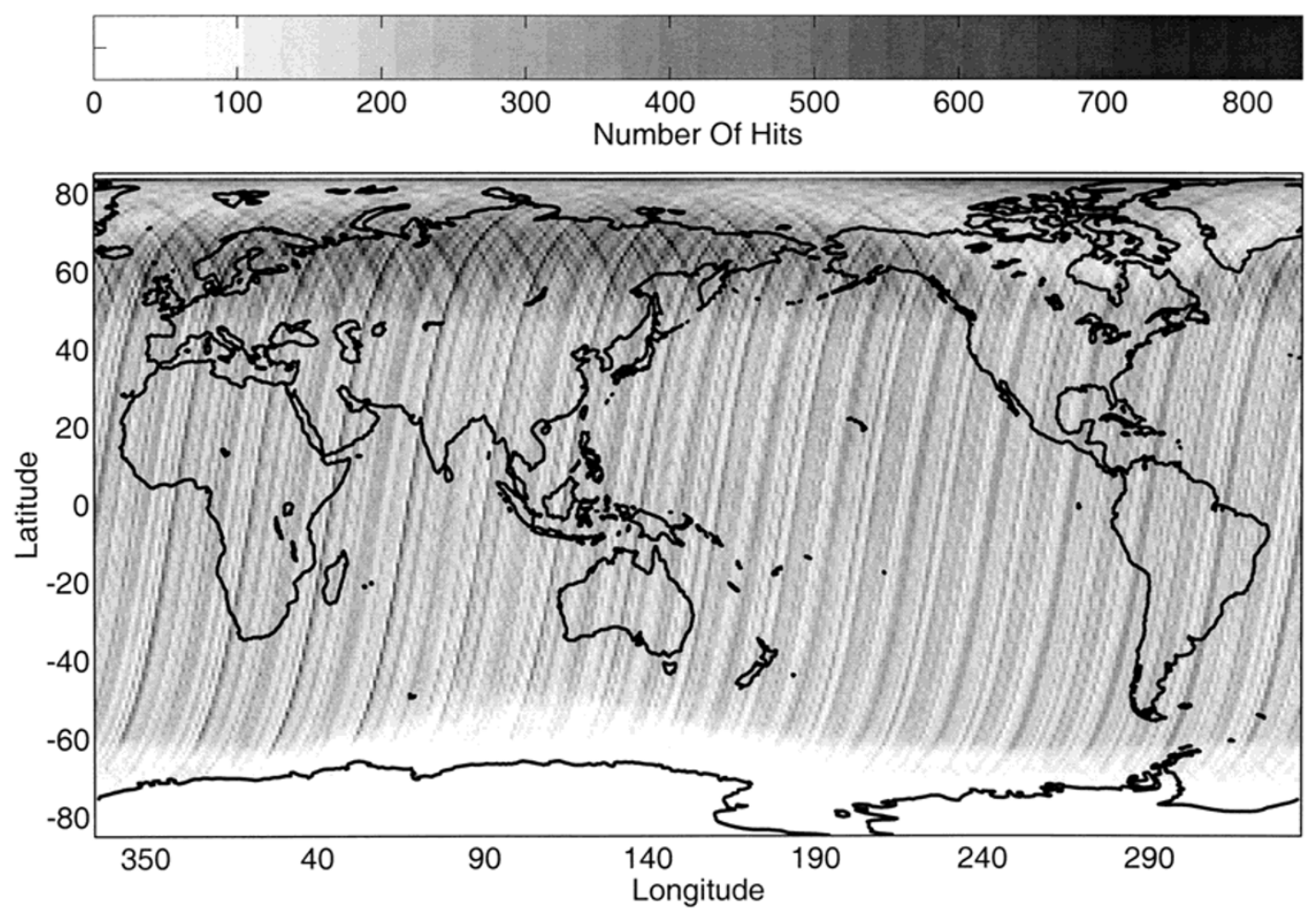

Fig. 3. Number of hits in each 1-degree by 1-degree bin, Magsat vector dawn data. 


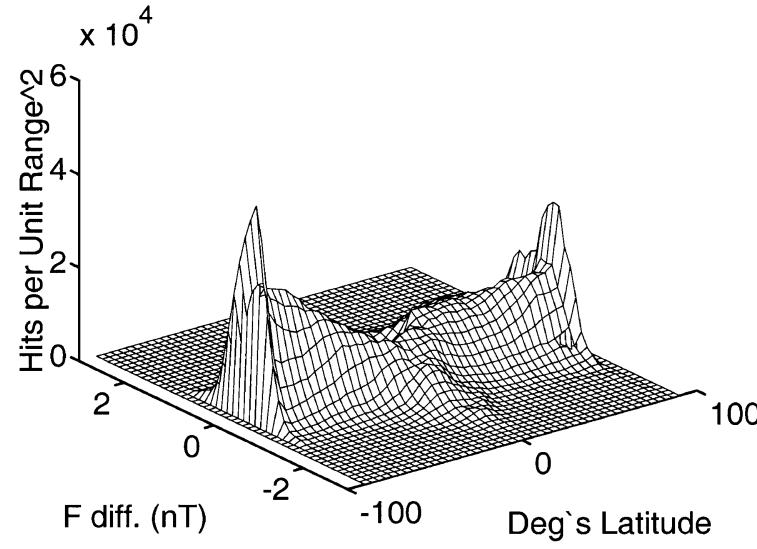

Fig. 4. Frequency distribution of the numerical difference between $\emptyset$ rsted total intensity values determined from vector (MAG-L) data and scalar (OVH) data.

erwise smooth contours of the scalar anomaly field averages, the furrows corresponding to sections of satellite orbits. The problem was alleviated by using a linear (or hyperplane) fit to the residuals to determine a mean scalar anomaly value at the centre of each bin at a sphere of radius $7130 \mathrm{~km}$ for Ørsted and $6800 \mathrm{~km}$ for Magsat. The hyperplane fit was found to be an effective method of altitude compensation and cell centring, reducing the radial distribution of a large amount of data to a relatively small amount of data evenly distributed over a sphere, suitable for high-degree spherical harmonic analysis and/or signal processing. The hyperplane fit is a linear approximation to the roughly exponential variation with altitude of crustal fields.

\section{Along-Track, High-Pass Filtering of Ørsted Scalar Data}

An along-track, high-pass filter, with a sharp cut-off below 14 cycles per satellite orbit has been used to remove the core field contribution from scalar data along each Ørsted satellite track. In the absence of theoretical expressions for the scalar field in terms of potential coefficients, one can only infer that some power from spherical harmonics of degree 13 or less in the potential will remain in the scalar field power of harmonics of 14 cycles per satellite orbit or higher. However, because the satellite tracks are almost north south, there will be very little power contributed to the cycles per satellite orbit from dependence on longitude, but there will be some power from core field zonal and low order terms. Therefore, the residuals remaining from the filtering process derive principally from the scalar anomaly field. By this means, there is an independent check on the distribution of Ørsted residuals over continental sized regions, without having to determine a core field potential model. The along-track filter method has the advantage that it works directly with satellite data and not with residuals. (The formulae for the Fourier decomposition of associated Legendre functions are given by Schuster (1903)).

The design of the high-pass filter takes into account that ripples in the stop band section of the filter response may cause leakage of zonal core field components into the filter output. Further, an insufficiently fast transition from stop

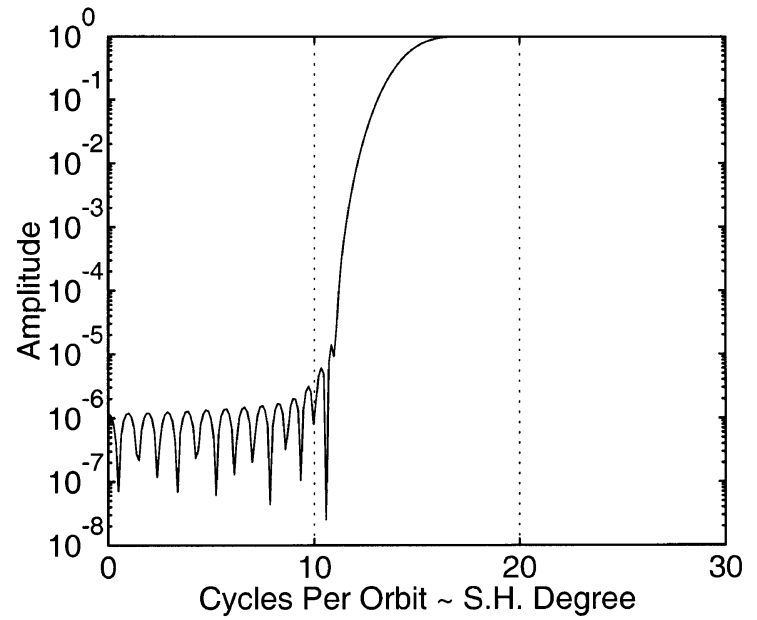

Fig. 5. Frequency response of the along-track high-pass filter used for the Ørsted OVH data.

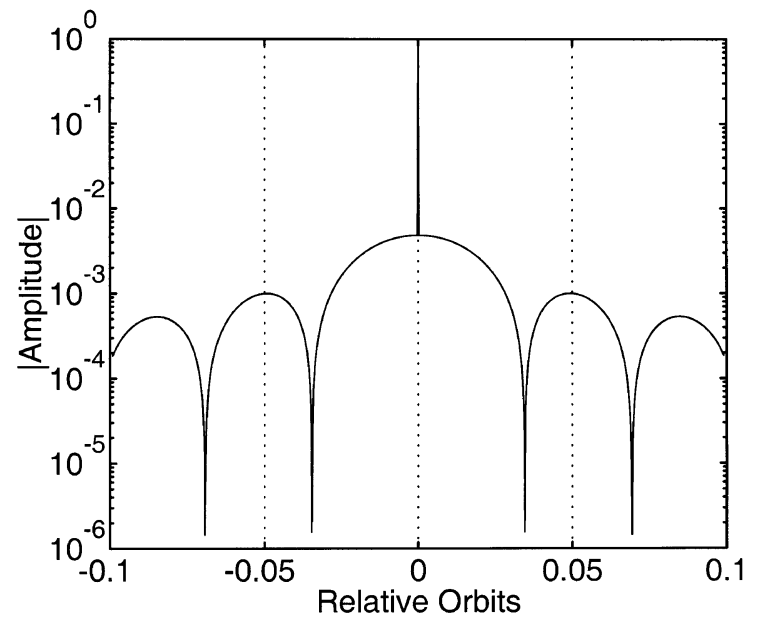

Fig. 6. The impulse response of the filter in Fig. 5.

band to pass band will cause a similar effect. Numerical results confirmed the difficulty in filtering out high-degree core field zonal terms, and therefore along-track high-pass filter results are confined to continental sized regions only.

The along-track filter was applied to the eight months of OVH scalar data between March and December 1999. All satellite scalar data were filtered (no $K p$ index was provided with this data set), however, any values in the filter output that were greater than $50 \mathrm{nT}$ were excluded from further analysis and did not go forward into the binning process.

Using simple non-recursive filters with a large sample sequence can alleviate some of these problems. Although recursive elliptic filtering was examined, there appears to be a problem when the stop band is a small fraction of the sampling frequency. The use of non-recursive filters allows one to use the fast Fourier transform convolution theorem to greatly reduce the amount of filtering computation required.

In the core field spherical harmonic potential series, the magnitude of the dipole (degree 1) term is about 30,000 nT, whilst that of the terms of degree 13 is on average about 


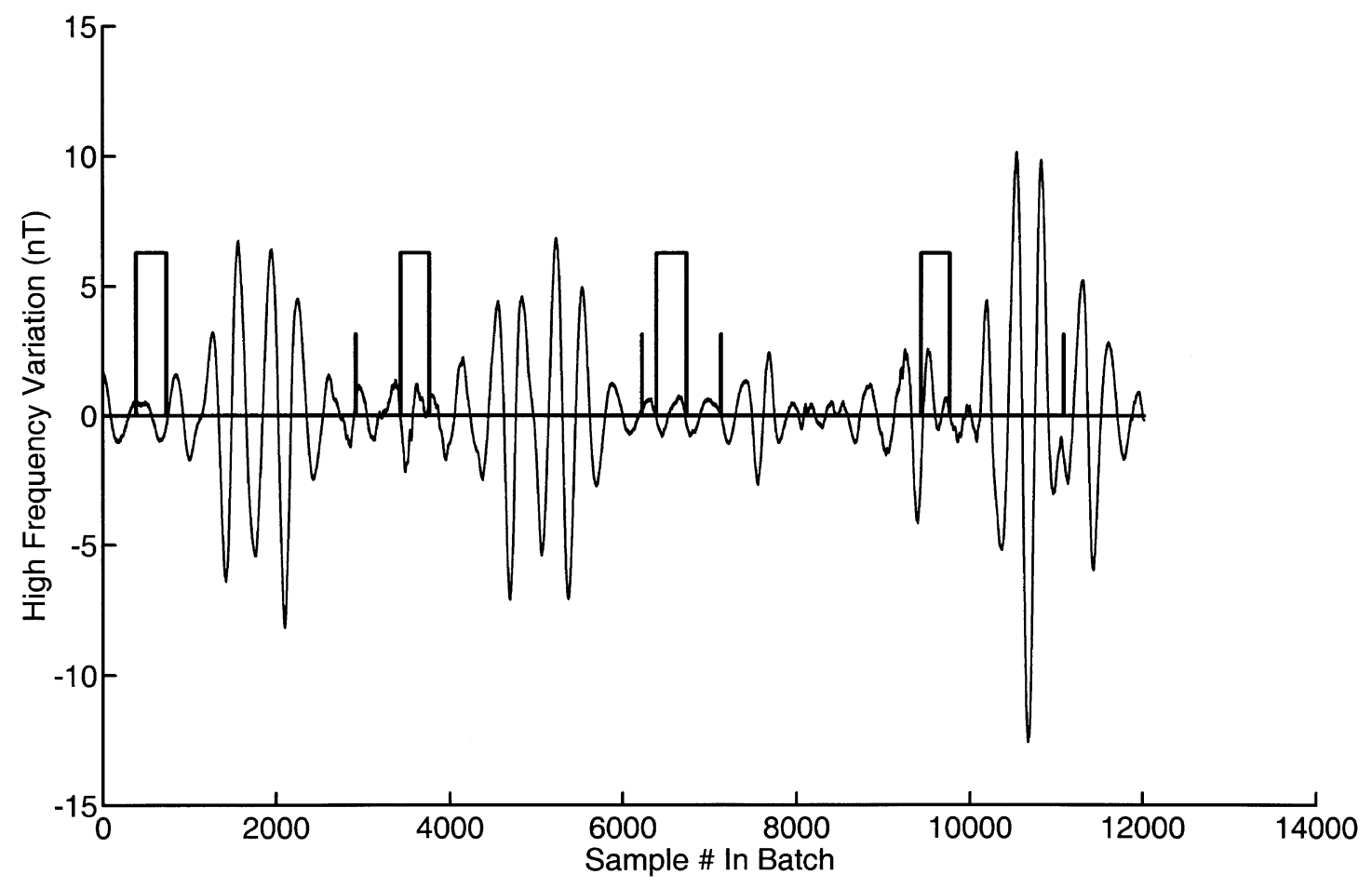

Fig. 7. Typical output from the along-track, high-pass filter used for Ørsted OVH data. Note that the interpolation flags are $1 / 2$ the height of the $\pm 10 \mathrm{deg}$ warning gate.

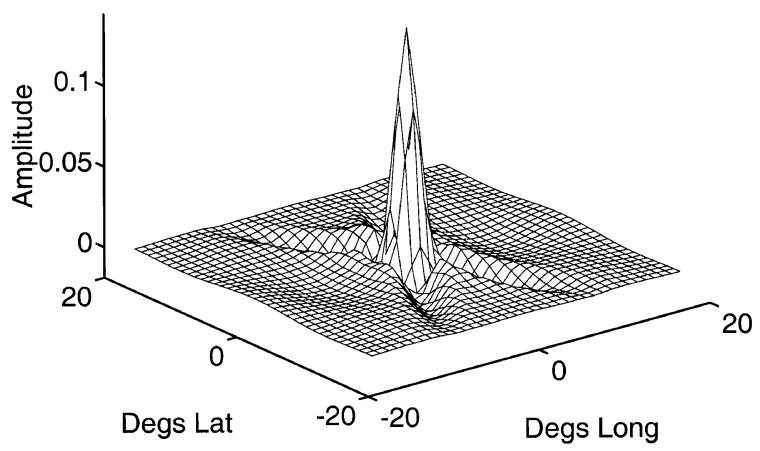

(a)

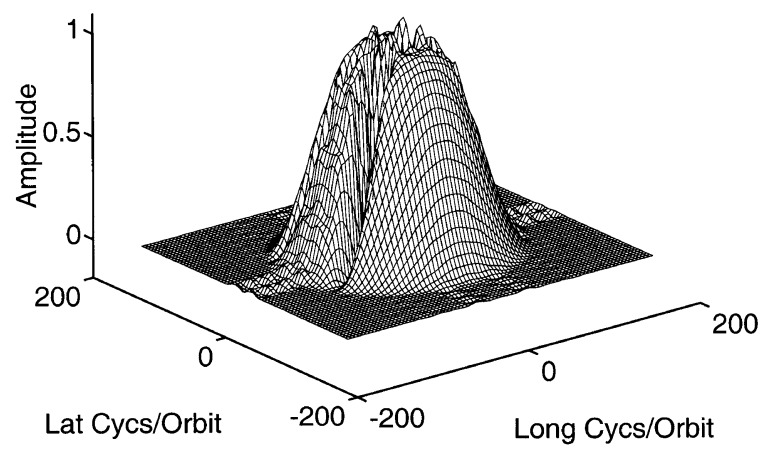

(b)

Fig. 8. (a) Real part of notch filter function. (b) Real part of notch filter response.

$1 \mathrm{nT}$. Translating this into an approximate Fourier expansion indicates the stop band ripple should be $50 \mathrm{~dB}$ below the pass band. The stop band ripple can be reduced using Kaiser (1974) weighting on the filter coefficients at the expense of making the transition from stop to pass band less steep.

A process of experimentation showed that a suitable filter could be achieved with a length of around 6000 samples using a Kaiser weighting parameter of 12 . Taking into account the sampling rate of the OVH data and the satellite speed this translates into a physical length of about an orbit. Figures 5 and 6 below show the essential filter characteristics, frequency and impulse response on a logarithmic scale.

Filtering was carried out in batches of three filter sample lengths that overlapped each other by a half-filter length. The half-filter lengths from either end of a batch are dis- carded because they contained spatial aliasing effects caused by circular convolution, and the remaining portions stored on file where they form the input to the binning procedure.

Figure 7 shows the contents of a typical batch with the half-filter ends removed. Included in the plot are warning flags showing:

a) where data missing from the $\mathrm{OVH}$ input sequence has been linearly interpolated from the valid data on either side (filter output coinciding with interpolated input was not stored for binning),

b) the $-10 \mathrm{deg}$ to $10 \mathrm{deg}$ latitude warning gate to highlight the position of the geographic equator. This approximates the position of the magnetic equator and aids visual inspection of the filtered output in the electrojet 

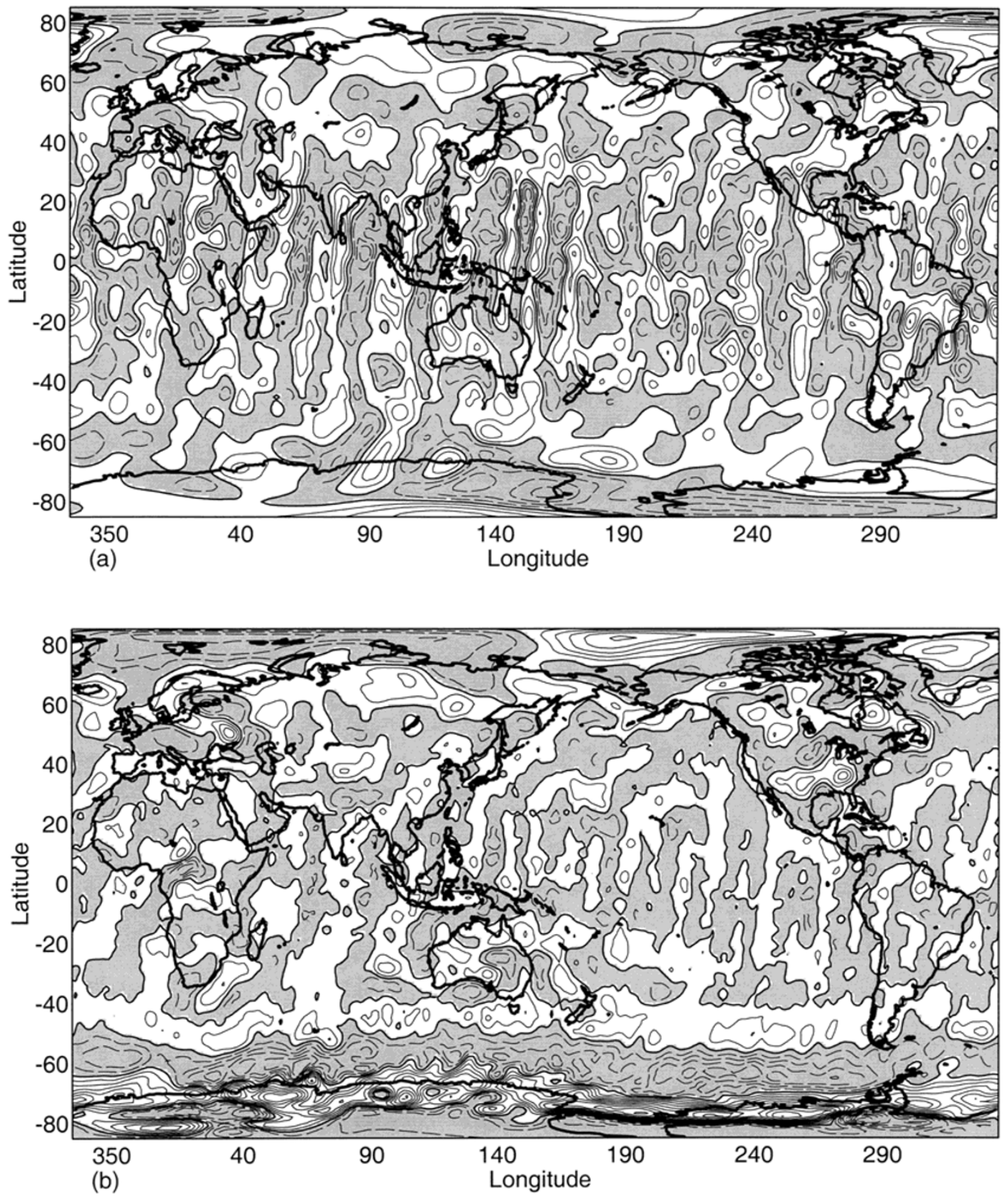

Fig. 9. (a) Global map of Ørsted scalar anomaly field from filtered residuals after core field subtraction, at $7130 \mathrm{~km}$, drawn from regional maps over continental sized regions. Contours $2 \mathrm{nT}$ intervals. (b) Global map of Magsat scalar anomaly field from filtered residuals after core field subtraction, at $6800 \mathrm{~km}$, drawn from regional maps over continental regions. Contours $4 \mathrm{nT}$ intervals.

region.

As can be seen from Fig. 7, the average magnitude of the filter output is about $5 \mathrm{nT}$, which is within the expected range of the scalar anomaly field.

\section{Spatial Filtering of Binned Ørsted Residuals}

After the binning, altitude compensation and cell centring processes, the contours of vector residuals still appeared to have furrows following sections of satellite tracks superimposed on the contours. Notch filters relying on the satellite track sections being almost straight and parallel were applied to further remove this over low latitude regions. The additive furrow signal appears as a straight line of peaks on the as- sociated two-dimensional Fast Fourier Transform (FFT) of such a regional plot. It is then a simple matter to filter out the tracks by a notch filter, designed by setting all the FFT coefficients near the lines to be zero. See Fig. 8 .

Notch filtering is not feasible and not used for regions with latitude above $45 \mathrm{deg}$ where the satellite tracks are more curved when plotted with east longitude as abscissa and colatitude as ordinate. A better method would be to determine the effect of the furrows on the spherical harmonic spectrum, and then to de-track on a global basis. No simple solution using this method is available at present. It is of course possible to go further and incorporate high and low pass spatial filtering with the notch filtering. 
To avoid end effects caused by the equivalent two-dimensional spatial convolution, all regional plots are filtered after first spatially limiting the filter convolution function to \pm 18 deg in longitude and latitude deg, and incorporating an 18 deg margin around each edge. This is removed after filtering and before the plots are generated.

From the core field and the filtered vector residual known in each bin, a scalar field is determined from the core field plus vector residual field and also for the core field alone. The difference between these scalar fields is then the scalar residual field. A global contour map of the scalar residual field is given for the Ørsted satellite, with $2 \mathrm{nT}$ contours, on a sphere of radius $7130 \mathrm{~km}$ in Fig. 9(a). A global contour map of the scalar residual field for the Magsat satellite, with $4 \mathrm{nT}$ contours, on a sphere of radius of $6800 \mathrm{~km}$ in Fig. 9(b). These maps include the zero contour, and negative values have been shaded in grey. At first glance they appear different to those of Arkani-Hamed et al. (1994), but a close inspection will show that they have many features in common.

The maps shown in Figs. 9(a) and 9(b) are composites of smaller regional analyses, such as those given below in Figs. 11, 12, and 13.

\section{Magsat Core Field Analysis}

Iterative spherical harmonic analyses were carried on six monthly vector data sets of dawn values, evaluating residuals at each step, and progressively rejecting those data with residuals greater than a chosen value. At the final stage, data were rejected with a residual value of $50 \mathrm{nT}$, and then final monthly sets of spherical harmonic coefficients were determined. The spherical harmonic coefficients were determined to degree and order 13 for internal terms and degree and order 1 for external terms. Only those data with $K p<2$ were used. The residuals were binned and interpolated to the centre of each bin on a sphere of radius $6800 \mathrm{~km}$. Notch filtering was applied to the spherical distribution of residuals.

\section{Magsat Vector Residual Analysis and Upward Continuation}

To allow a direct comparison between the results of the Ørsted subtractive model, the Ørsted along-track high-pass filtering, and the Magsat subtractive model, an internal spherical harmonic model of the processed Magsat vector residuals up to degree and order 160 has been produced to be upward continued to the Ørsted level. Degree and order 160 is much greater than the degree and order $n=63$ used by Cain et al. (1989), but is in agreement with the conclusion of Wang (1987), that the Magsat data contain information up to the limit $n=160$. Truncation at a lower degree and order than $n=160$ gave an unwanted, over-smoothed appearance to the contours. A Lowes spectrum is given in Fig. 10. Although downward continuation from satellite altitude is numerically unstable, characterized by a minimum altitude below which the spherical harmonic spectrum does not converge, the same is not true of upward continuation.

The altitude compensated, cell centred, binned Magsat vector residuals are now reduced to three values in each 1 degree by 1 degree bin evenly distributed over the surface of a sphere. The vector residual components are the standard local Cartesian components, and they were converted to

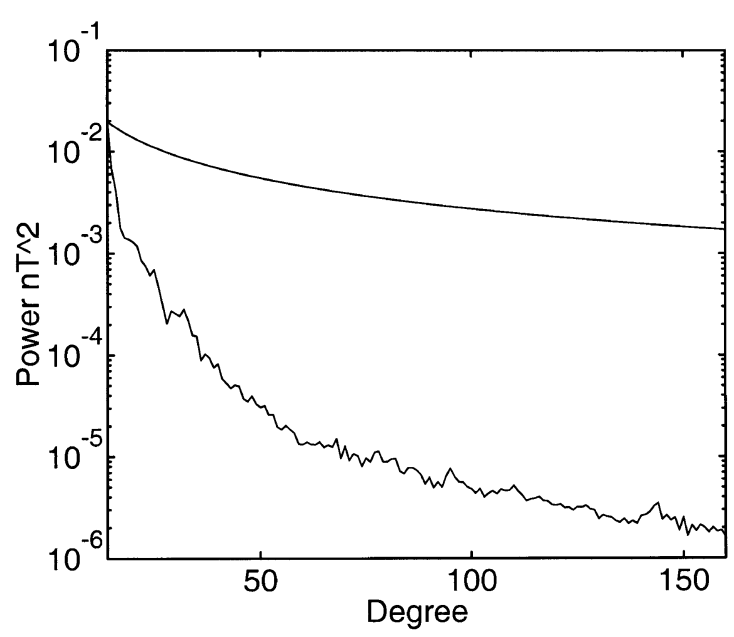

Fig. 10. Lowes spectrum of Magsat residual spherical harmonic analysis, degree 14 to 160 .

geocentric global Cartesian components. Each of the three global Cartesian components was represented by a linear combination of surface spherical harmonics. The orthogonality of surface spherical harmonics over a sphere extends to the three vector surface spherical harmonics over a sphere, corresponding to internal, external, and non-potential fields. The coefficients of the internal, external, and non-potential fields can be determined independently without the need for further spherical harmonic analysis, e.g., Winch (1968). Linear combinations of three spherical harmonic coefficients from each component provide independent coefficients for the internal, external, and non-potential fields. In this case, namely, spherical harmonic analysis of residual vector fields, only the internal field is required, and the coefficients do not change if the external and/or non-potential fields are determined.

Combining the potentials of the core field model and the internal residual model a scalar main field was determined at the normalized Ørsted radius of $7130 \mathrm{~km}$. A second scalar field was determined using only the core field model. The difference between these two scalar fields gave the Magsat scalar anomaly field at the normalized Ørsted radius.

Pass- and notch-filtering were applied to the Magsat scalar anomaly field. The Ørsted along-track filtered scalar residuals were treated in exactly the same way as the Magsat scalar anomaly field.

\section{Scalar Anomaly Regional Studies}

Graphical output of the scalar anomaly fields over three regions is given in the following figures: Continental Australia, Fig. 11; Northern Europe, Fig. 12; North America, Fig. 13.

Note that the global scalar anomaly fields in Fig. 9(a) have been made by combining anomaly fields from continental sized regions, such as those given in Figs. 11(b), 12(b), and 13(b). Similarly, Fig. 9(b) combines fields such as those shown in Figs. 11(c), 12(c), and 13(c).

Notch-, high- and low-pass filtering is applied to all of Figs. 11, 12, and 13, (a), (b), (c), and (d). More high frequencies were removed from Figs. 11(b), 12(b), and 13(b) 

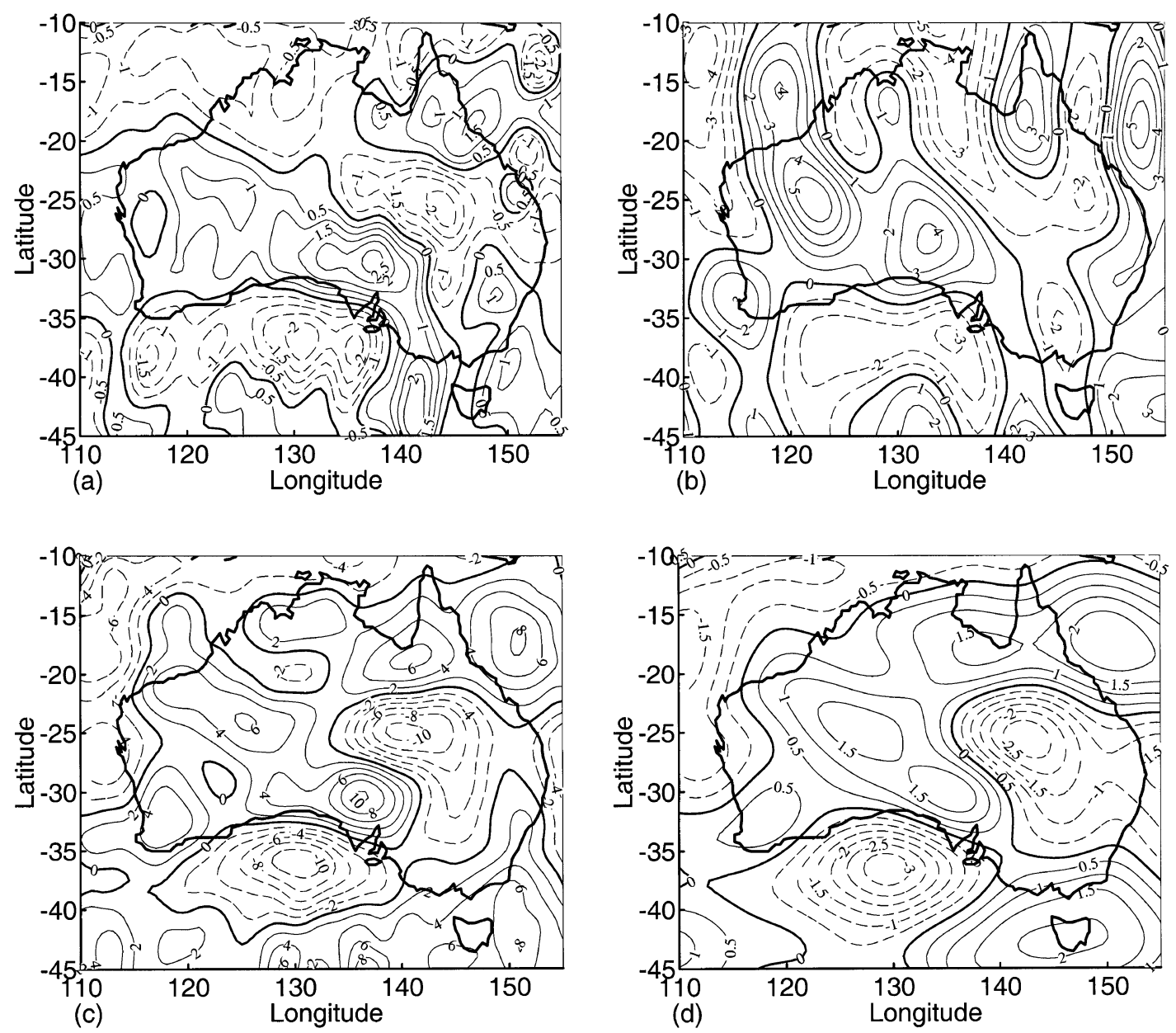

Fig. 11. (a) Ørsted scalar anomaly field at $7130 \mathrm{~km}$ when core field removed by along-track, high-pass filtering. (b) Ørsted crustal anomal field at 7130 $\mathrm{km}$ when core field removed by degree and order 13 model. (c) Magsat scalar anomaly at $6800 \mathrm{~km}$ from dawn data when core field removed by degree and order 13 model. (d) Magsat scalar anomaly field of Fig. 11(c) upward continued to $7130 \mathrm{~km}$.

because of sparse data distributions.

\subsection{The Australian continent}

Figure 11(a) shows the residuals determined from alongtrack, high-pass filtering of the Ørsted OVH scalar data. A series of minima of about $-2 \mathrm{nT}$ appear over the Great Australian Bight, a maximum of $2.5 \mathrm{nT}$ over Lake Eyre in South Australia west of Broken Hill, extending to another maximum of $1 \mathrm{nT}$ from Lake Carnegie to the Hamersley range in Western Australia. There is a minimum of $2 \mathrm{nT}$ over Longreach in the Great Artesian Basin, and a maximum of 1.5 nT over Townsville on the Queensland Coast. Figure 11(c) shows scalar field anomalies determined after subtracting the degree and order 13 core field monthly models from Magsat dawn vector data, at a median radius of $6800 \mathrm{~km}$. These Magsat residuals have then been upward continued to Ørsted median radius of $7130 \mathrm{~km}$, and the results shown in Fig. 11(d) are in good agreement with the results from along-track high pass filtering shown in Fig. 11(a).

Figure 11(b) shows Ørsted residuals after subtracting degree and order 13 monthly models calculated by iterative analyses of vector and scalar nighttime data. The residuals were determined from vector data only and then heavily smoothed by removing a greater proportion of the high spatial frequencies than for Magsat (and Ørsted along-track filter- ing) via the combined pass- and notch-filter. This was to reduce noise caused by the relative sparseness of the vector data. The 2 nT minimum over Longreach in Fig. 11(a) does not appear at all in Fig. 11(b).

For the Australian region it would appear that along-track high-pass filtering is able to produce scalar anomaly fields from Ørsted data compatible with known results from Magsat. Core field models to degree and order 13 based on nighttime Ørsted vector and scalar data give residuals which are only marginally compatible with the corresponding Magsat results for the Australian region.

\subsection{Scandinavian and Kursk anomalies}

Figure 12(a) shows contoured residuals from along-track, high-pass filtering of the Ørsted OVH scalar data. There is a maximum scalar anomaly residual of $2.5 \mathrm{nT}$ over Kursk in the Ukraine extending westwards to another maximum of $3.5 \mathrm{nT}$ over the Polish and Lithuanian coast. There is also a minimum of $-4 \mathrm{nT}$ over the Gulf of Bothnia. A zonal extended residual maximum of $3.5 \mathrm{nT}$ is seen passing between Norway and Svalbard, possibly associated with the auroral current system, or possibly with the leakage of zonal terms in the core field potential through the high-pass filter.

Figure 12(b) shows the residuals determined after subtracting a degree and order 13 core field models from the MAG-L 

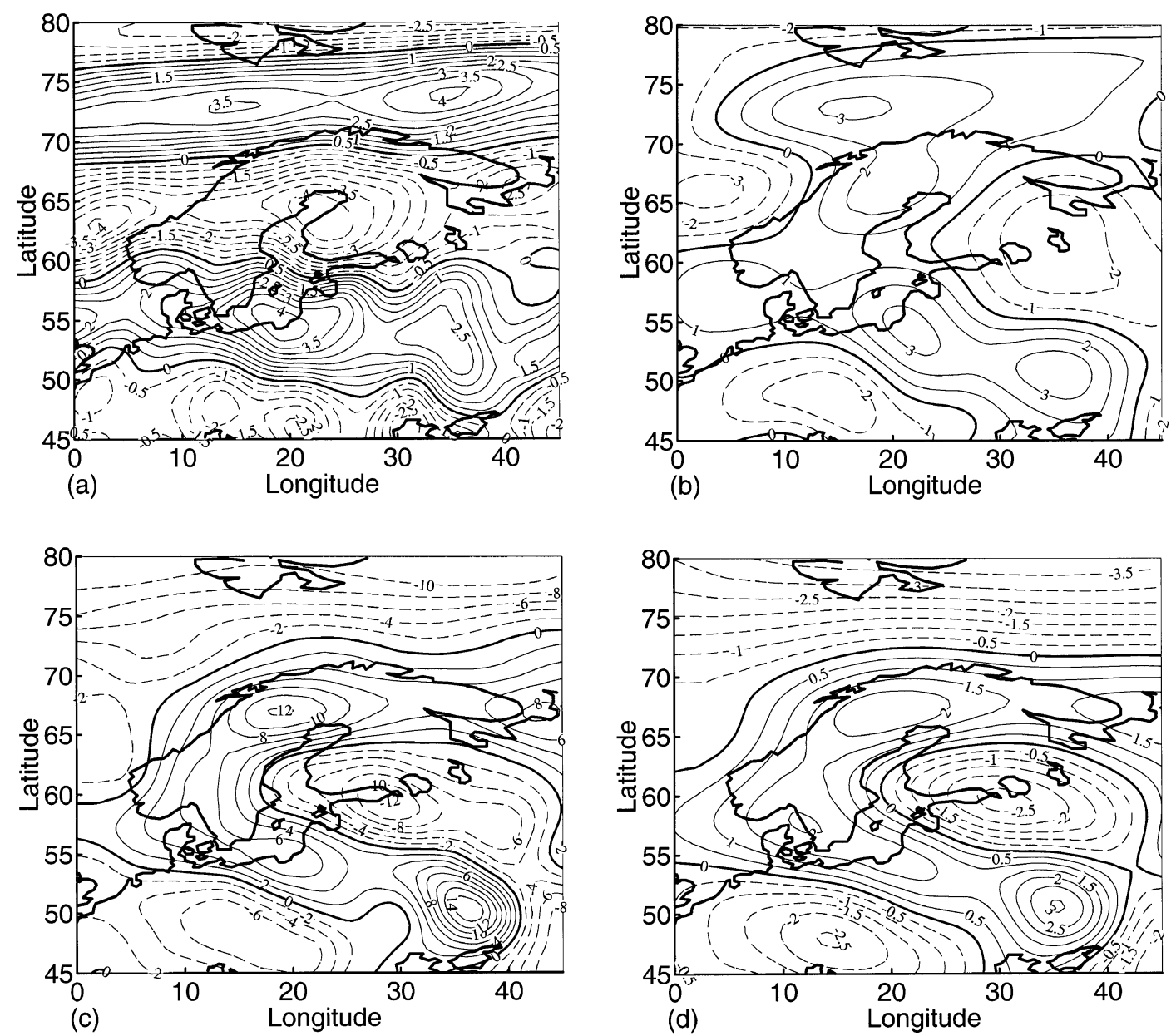

Fig. 12. (a) Ørsted scalar anomaly field at $7130 \mathrm{~km}$ when core field removed by along-track, high-pass filtering. (b) Ørsted scalar anomal field at $7130 \mathrm{~km}$ when core field removed by degree and order 13 model. (c) Magsat scalar anomaly at $6800 \mathrm{~km}$ from dawn data when core field removed by degree and order 13 model. (d) Magsat scalar anomaly field of Fig. 12(c) upward continued to $7130 \mathrm{~km}$.

vector observations. The Kursk anomaly is $3 \mathrm{nT}$, extending westwards to a 3 nT maximum over the Polish and Lithuanian coast. A minimum of $-2 \mathrm{nT}$ appears centred on Lake Onega, with a $3 \mathrm{nT}$ anomaly between Norway and Svalbard extending southwards toward northern Sweden.

Figure 12(c) shows the residuals as determined at the Magsat normalized radius of $6800 \mathrm{~km}$. The Kursk maximum is $14 \mathrm{nT}$, and extending westwards to the Polish coast. There is a minimum of $-12 \mathrm{nT}$ over Leningrad. The Kiruna anomaly is a well-defined maximum of $12 \mathrm{nT}$.

When continued upwards to the normalized Ørsted radius of $7130 \mathrm{~km}$, as in Fig. 12(d), the Kursk anomaly is reduced to $3 \mathrm{nT}$, ridging westwards. The Kiruna anomaly is $2 \mathrm{nT}$. There is a well-defined minimum of $-2.5 \mathrm{nT}$ centred on Leningrad.

It appears that the upward continued Magsat map Fig. 12(d) is better correlated with the residuals obtained after subtracting a core field model of degree and order 13, as in Fig. 12(b) than with the zonal appearance of the alongtrack high-pass filtered Ørsted data Fig. 12(a).

\subsection{North American anomalies}

Figure 13(a) shows the results from along-track, high-pass filtering of the Ørsted OVH scalar data. A positive region over most of the United States has a series of maxima from the West coast to the East coast. This positive region cor- responds to a similar positive region shown in Fig. 13(d) for the upward continued Magsat residuals, with maxima of $2.5 \mathrm{nT}$ and $3.5 \mathrm{nT}$ over Colorado and Kentucky respectively. Figure 13(a) shows a zonal band of negative values across the southern United States and Northern Mexico with minima of $-2.5 \mathrm{nT}$, over Houston, $-3 \mathrm{nT}$ over Galveston and $-1.5 \mathrm{nT}$ north of the Bahamas, corresponding to the region of negative values with a minimum of $-3 \mathrm{nT}$ centred in the Gulf of Mexico in Fig. 13(d). Figure 13(a) shows a region of positive residuals in Southern Mexico and the Yucatan peninsula extending across to Cuba, although in Fig. 13(d), the positive residual region has been split by the region of negative residuals in the Gulf of Mexico joining up with a negative residual region over the Caribbean. Figure 13(b), the Ørsted residuals determined from subtraction of a degree and order 13 core field model, show the same principal features as in Figs. 13(a) and 13(d). There are strong minima of $-4 \mathrm{nT}$ over the Gulf of Mexico and the Sierra Madre. Positive regions appear over southern Mexico, Yucatan and Cuba and Haiti. A positive region with a maximum of $4 \mathrm{nT}$ over New Mexico ridges eastward to a $3 \mathrm{nT}$ maximum over Kentucky. Again the agreement seems to be better between the upward continued Magsat residual contours in Fig. 13(a) and the Ørsted residuals obtained by along-track high-pass 
Table 1. Spherical harmonic coefficients from Ørsted data for the core field to degree and order 13, for the months of 1999 as indicated.

\begin{tabular}{|c|c|c|c|c|}
\hline \multicolumn{5}{|c|}{ Ørsted spherical harmonic coefficients } \\
\hline$g / h(n, m)$ & April & May & September & December \\
\hline$H(1,1)$ & -4.5 & -4.9 & -8.9 & -4.4 \\
\hline$G(1,1)$ & 4.2 & 3.3 & 5.9 & 3.4 \\
\hline$G(1,0)$ & 39.9 & 33.3 & 65.5 & 36.5 \\
\hline$g(1,0)$ & -29646.0 & -29642.3 & -29648.9 & -29633.5 \\
\hline$g(1,1)$ & -1737.3 & -1737.3 & -1733.5 & -1731.1 \\
\hline$h(1,1)$ & 5203.1 & 5202.1 & 5195.6 & 5189.5 \\
\hline$g(2,0)$ & -2257.0 & -2259.3 & -2264.3 & -2266.8 \\
\hline$g(2,1)$ & 3070.3 & 3069.4 & 3069.0 & 3068.5 \\
\hline$h(2,1)$ & -2466.1 & -2467.4 & -2475.8 & -2481.9 \\
\hline$g(2,2)$ & 1672.7 & 1672.9 & 1672.0 & 1670.4 \\
\hline$h(2,2)$ & -451.7 & -451.6 & -455.1 & -456.7 \\
\hline$g(3,0)$ & 1337.1 & 1337.5 & 1339.6 & 1338.3 \\
\hline$g(3,1)$ & -2284.9 & -2285.0 & -2286.8 & -2287.5 \\
\hline$h(3,1)$ & -229.4 & -230.2 & -228.9 & -227.4 \\
\hline$g(3,2)$ & 1252.8 & 1253.0 & 1252.6 & 1252.2 \\
\hline$h(3,2)$ & 295.9 & 295.9 & 294.0 & 293.2 \\
\hline$g(3,3)$ & 720.2 & 720.1 & 717.0 & 714.5 \\
\hline$h(3,3)$ & -484.1 & -484.4 & -488.7 & -490.8 \\
\hline$g(4,0)$ & 934.1 & 934.6 & 933.0 & 932.5 \\
\hline$g(4,1)$ & 786.3 & 786.5 & 787.7 & 786.3 \\
\hline$h(4,1)$ & 271.3 & 271.2 & 272.8 & 273.1 \\
\hline$g(4,2)$ & 256.2 & 255.6 & 252.4 & 250.3 \\
\hline$h(4,2)$ & -232.8 & -232.5 & -232.1 & -231.9 \\
\hline$g(4,3)$ & -405.5 & -405.4 & -404.7 & -403.5 \\
\hline$h(4,3)$ & 116.3 & 116.8 & 117.8 & 119.2 \\
\hline$g(4,4)$ & 112.4 & 112.5 & 111.7 & 111.6 \\
\hline$h(4,4)$ & -303.2 & -303.5 & -303.5 & -303.5 \\
\hline$g(5,0)$ & -218.2 & -217.8 & -218.8 & -218.3 \\
\hline$g(5,1)$ & 350.3 & 350.1 & 350.8 & 351.4 \\
\hline$h(5,1)$ & 44.8 & 44.6 & 44.9 & 43.6 \\
\hline$g(5,2)$ & 224.3 & 223.6 & 222.5 & 221.8 \\
\hline$h(5,2)$ & 171.4 & 170.8 & 171.9 & 172.0 \\
\hline$g(5,3)$ & -129.0 & -129.5 & -130.4 & -130.4 \\
\hline$h(5,3)$ & -134.5 & -134.2 & -133.6 & -133.1 \\
\hline$g(5,4)$ & -168.5 & -168.5 & -168.3 & -168.9 \\
\hline$h(5,4)$ & -42.5 & -41.7 & -40.4 & -39.6 \\
\hline$g(5,5)$ & -12.9 & -13.0 & -12.7 & -13.0 \\
\hline$h(5,5)$ & 106.9 & 106.5 & 106.1 & 106.1 \\
\hline$g(6,0)$ & 70.9 & 71.3 & 70.9 & 71.1 \\
\hline$g(6,1)$ & 68.6 & 68.1 & 68.5 & 68.2 \\
\hline$h(6,1)$ & -16.7 & -16.7 & -16.6 & -17.3 \\
\hline$g(6,2)$ & 73.7 & 74.0 & 74.6 & 74.4 \\
\hline$h(6,2)$ & 64.6 & 64.6 & 63.9 & 63.7 \\
\hline$g(6,3)$ & -162.4 & -162.1 & -161.3 & -160.6 \\
\hline$h(6,3)$ & 64.9 & 65.1 & 65.3 & 65.4 \\
\hline$g(6,4)$ & -4.7 & -4.9 & -5.5 & -5.6 \\
\hline$h(6,4)$ & -61.0 & -60.7 & -60.9 & -61.2 \\
\hline$g(6,5)$ & 17.0 & 17.1 & 16.4 & 16.8 \\
\hline$h(6,5)$ & 0.9 & 0.8 & 0.8 & 0.9 \\
\hline$g(6,6)$ & -90.6 & -90.8 & -90.9 & -90.4 \\
\hline
\end{tabular}

\begin{tabular}{|c|c|c|c|c|}
\hline \multicolumn{5}{|c|}{ Ørsted spherical harmonic coefficients } \\
\hline$g / h(n, m)$ & April & May & September & December \\
\hline$h(6,6)$ & 42.6 & 42.6 & 43.7 & 43.7 \\
\hline$g(7,0)$ & 78.2 & 78.4 & 78.8 & 77.9 \\
\hline$g(7,1)$ & -74.3 & -74.5 & -74.9 & -74.5 \\
\hline$h(7,1)$ & -64.7 & -64.8 & -65.1 & -64.1 \\
\hline$g(7,2)$ & 0.5 & 0.1 & -0.5 & -0.2 \\
\hline$h(7,2)$ & -23.8 & -23.9 & -24.0 & -24.5 \\
\hline$g(7,3)$ & 32.9 & 32.8 & 33.2 & 32.9 \\
\hline$h(7,3)$ & 5.9 & 6.2 & 6.1 & 6.2 \\
\hline$g(7,4)$ & 8.6 & 8.5 & 8.9 & 9.3 \\
\hline$h(7,4)$ & 23.9 & 23.9 & 24.0 & 24.0 \\
\hline$g(7,5)$ & 6.4 & 6.7 & 6.5 & 6.8 \\
\hline$h(7,5)$ & 15.1 & 15.1 & 14.7 & 14.7 \\
\hline$g(7,6)$ & 7.3 & 7.7 & 7.2 & 7.3 \\
\hline$h(7,6)$ & -25.4 & -25.3 & -25.4 & -25.1 \\
\hline$g(7,7)$ & -1.8 & -1.4 & -1.2 & -1.1 \\
\hline$h(7,7)$ & -6.3 & -5.6 & -5.6 & -5.7 \\
\hline$g(8,0)$ & 23.6 & 24.6 & 24.5 & 24.5 \\
\hline$g(8,1)$ & 7.2 & 6.8 & 7.3 & 6.6 \\
\hline$h(8,1)$ & 12.1 & 11.6 & 12.0 & 12.0 \\
\hline$g(8,2)$ & -8.4 & -8.4 & -8.3 & -8.9 \\
\hline$h(8,2)$ & -21.8 & -21.8 & -22.0 & -21.1 \\
\hline$g(8,3)$ & -8.0 & -7.8 & -7.8 & -7.8 \\
\hline$h(8,3)$ & 8.1 & 8.1 & 7.9 & 8.3 \\
\hline$g(8,4)$ & -16.5 & -16.5 & -16.6 & -16.9 \\
\hline$h(8,4)$ & -21.6 & -21.8 & -21.7 & -21.4 \\
\hline$g(8,5)$ & 8.9 & 8.9 & 9.1 & 9.2 \\
\hline$h(8,5)$ & 15.4 & 15.5 & 15.2 & 15.5 \\
\hline$g(8,6)$ & 6.8 & 6.7 & 7.0 & 6.9 \\
\hline$h(8,6)$ & 9.0 & 9.2 & 8.9 & 8.8 \\
\hline$g(8,7)$ & -7.6 & -7.7 & -7.6 & -7.9 \\
\hline$h(8,7)$ & -15.1 & -15.2 & -15.2 & -15.1 \\
\hline$g(8,8)$ & -7.4 & -7.2 & -7.1 & -6.9 \\
\hline$h(8,8)$ & -2.5 & -2.1 & -2.1 & -2.1 \\
\hline$g(9,0)$ & 5.8 & 5.9 & 5.4 & 3.8 \\
\hline$g(9,1)$ & 9.1 & 8.6 & 8.7 & 9.2 \\
\hline$h(9,1)$ & -20.9 & -20.5 & -20.1 & -19.4 \\
\hline$g(9,2)$ & 2.7 & 2.5 & 2.5 & 2.5 \\
\hline$h(9,2)$ & 13.7 & 13.5 & 13.9 & 13.7 \\
\hline$g(9,3)$ & -8.3 & -8.6 & -8.3 & -8.3 \\
\hline$h(9,3)$ & 12.3 & 12.6 & 12.6 & 12.8 \\
\hline$g(9,4)$ & 6.5 & 6.5 & 6.5 & 6.3 \\
\hline$h(9,4)$ & -6.5 & -6.2 & -6.3 & -6.5 \\
\hline$g(9,5)$ & -8.7 & -8.7 & -8.8 & -8.9 \\
\hline$h(9,5)$ & -8.4 & -8.2 & -8.5 & -8.3 \\
\hline$g(9,6)$ & -1.4 & -1.6 & -1.6 & -1.5 \\
\hline$h(9,6)$ & 8.5 & 8.3 & 8.2 & 8.5 \\
\hline$g(9,7)$ & 9.4 & 9.0 & 9.1 & 9.1 \\
\hline$h(9,7)$ & 4.1 & 3.9 & 3.8 & 3.9 \\
\hline$g(9,8)$ & -3.9 & -4.1 & -4.3 & -4.2 \\
\hline$h(9,8)$ & -8.0 & -8.2 & -8.2 & -8.3 \\
\hline
\end{tabular}


Table 1. (continued)

\begin{tabular}{|c|c|c|c|c|}
\hline \multicolumn{5}{|c|}{ Ørsted spherical harmonic coefficients } \\
\hline$g / h(n, m)$ & April & May & September & December \\
\hline$g(9,9)$ & -8.3 & -8.1 & -7.9 & -8.1 \\
\hline$h(9,9)$ & 4.4 & 4.6 & 4.6 & 5.0 \\
\hline$g(10,0)$ & -2.5 & -2.2 & -2.4 & -3.2 \\
\hline$g(10,1)$ & -5.3 & -5.7 & -5.4 & -5.9 \\
\hline$h(10,1)$ & 1.8 & 0.9 & 0.9 & 2.0 \\
\hline$g(10,2)$ & 2.0 & 1.7 & 2.0 & 1.9 \\
\hline$h(10,2)$ & 0.2 & -0.1 & -0.7 & -0.7 \\
\hline$g(10,3)$ & -3.1 & -3.0 & -3.0 & -3.2 \\
\hline$h(10,3)$ & 3.9 & 3.8 & 3.7 & 3.8 \\
\hline$g(10,4)$ & -0.5 & -0.6 & -0.5 & -0.4 \\
\hline$h(10,4)$ & 4.9 & 4.9 & 4.9 & 5.0 \\
\hline$g(10,5)$ & 3.9 & 3.6 & 3.7 & 3.6 \\
\hline$h(10,5)$ & -5.7 & -5.9 & -5.7 & -5.9 \\
\hline$g(10,6)$ & 1.2 & 1.2 & 1.4 & 1.2 \\
\hline$h(10,6)$ & -1.2 & -1.3 & -1.2 & -1.0 \\
\hline$g(10,7)$ & 2.0 & 2.0 & 2.0 & 1.9 \\
\hline$h(10,7)$ & -2.8 & -2.8 & -2.7 & -2.9 \\
\hline$g(10,8)$ & 4.4 & 4.4 & 4.3 & 4.4 \\
\hline$h(10,8)$ & 0.3 & 0.2 & 0.2 & 0.4 \\
\hline$g(10,9)$ & 0.6 & 0.2 & 0.9 & 0.4 \\
\hline$h(10,9)$ & -2.2 & -1.9 & -2.4 & -2.0 \\
\hline$g(10,10)$ & -0.8 & -1.0 & -1.1 & -1.0 \\
\hline$h(10,10)$ & -7.5 & -7.8 & -7.4 & -7.7 \\
\hline$g(11,0)$ & 3.4 & 3.5 & 3.4 & 1.7 \\
\hline$g(11,1)$ & -1.8 & -2.1 & -2.3 & -2.5 \\
\hline$h(11,1)$ & -1.9 & -1.3 & -1.1 & 0.8 \\
\hline$g(11,2)$ & -2.9 & -2.7 & -2.8 & -2.0 \\
\hline$h(11,2)$ & 0.9 & 1.2 & 1.1 & 2.3 \\
\hline$g(11,3)$ & 1.4 & 1.4 & 1.6 & 1.8 \\
\hline$h(11,3)$ & -1.0 & -0.6 & -0.8 & -0.8 \\
\hline$g(11,4)$ & -0.2 & -0.1 & -0.1 & -0.3 \\
\hline$h(11,4)$ & -2.7 & -2.7 & -2.6 & -2.4 \\
\hline$g(11,5)$ & 0.0 & 0.1 & 0.0 & 0.2 \\
\hline$h(11,5)$ & 1.1 & 1.0 & 0.8 & 1.0 \\
\hline$g(11,6)$ & -0.7 & -0.6 & -0.6 & -0.9 \\
\hline$h(11,6)$ & -0.7 & -0.7 & -0.5 & -0.6 \\
\hline$g(11,7)$ & 0.7 & 0.8 & 0.6 & 0.8 \\
\hline$h(11,7)$ & -2.8 & -2.6 & -3.0 & -2.6 \\
\hline$g(11,8)$ & 1.6 & 1.6 & 1.9 & 1.6 \\
\hline$h(11,8)$ & -0.8 & -0.8 & -0.9 & -1.1 \\
\hline$g(11,9)$ & 0.2 & -0.1 & 0.6 & 0.0 \\
\hline$h(11,9)$ & -1.0 & -1.2 & -1.1 & -0.9 \\
\hline$g(11,10)$ & 1.0 & 1.7 & 0.8 & 1.0 \\
\hline$h(11,10)$ & -1.6 & -1.8 & -1.9 & -1.9 \\
\hline$g(11,11)$ & 4.2 & 3.9 & 4.3 & 4.2 \\
\hline$h(11,11)$ & -0.5 & -1.0 & -0.9 & -0.9 \\
\hline$g(12,0)$ & -1.5 & -1.5 & -1.8 & -2.7 \\
\hline$g(12,1)$ & 0.9 & 0.1 & 0.5 & 0.8 \\
\hline$h(12,1)$ & -0.9 & -1.7 & -1.7 & -1.1 \\
\hline$g(12,2)$ & 0.0 & -0.5 & -0.3 & 0.3 \\
\hline
\end{tabular}

\begin{tabular}{|c|c|c|c|c|}
\hline \multicolumn{5}{|c|}{ Ørsted spherical harmonic coefficients } \\
\hline$g / h(n, m)$ & April & May & September & December \\
\hline$h(12,2)$ & 0.6 & -0.1 & -0.7 & -0.7 \\
\hline$g(12,3)$ & 1.2 & 0.8 & 0.6 & 1.1 \\
\hline$h(12,3)$ & 3.0 & 3.0 & 2.8 & 2.3 \\
\hline$g(12,4)$ & 0.1 & 0.0 & -0.3 & -0.4 \\
\hline$h(12,4)$ & -2.5 & -2.7 & -2.3 & -2.8 \\
\hline$g(12,5)$ & 1.1 & 0.7 & 0.8 & 0.8 \\
\hline$h(12,5)$ & 0.6 & 0.6 & 0.6 & 0.7 \\
\hline$g(12,6)$ & -0.5 & -0.6 & -0.2 & -0.4 \\
\hline$h(12,6)$ & 0.2 & 0.4 & 0.4 & 0.3 \\
\hline$g(12,7)$ & 0.3 & 0.2 & 0.4 & 0.1 \\
\hline$h(12,7)$ & 0.0 & 0.0 & -0.2 & 0.0 \\
\hline$g(12,8)$ & -0.3 & -0.4 & -0.2 & -0.4 \\
\hline$h(12,8)$ & 0.0 & -0.1 & 0.2 & 0.0 \\
\hline$g(12,9)$ & -0.4 & -0.2 & -0.6 & -0.4 \\
\hline$h(12,9)$ & 0.5 & 0.4 & 0.5 & 0.3 \\
\hline$g(12,10)$ & -0.7 & 0.0 & -0.2 & -0.2 \\
\hline$h(12,10)$ & -1.2 & -0.7 & -1.0 & -1.0 \\
\hline$g(12,11)$ & -0.1 & -0.1 & 0.2 & 0.1 \\
\hline$h(12,11)$ & -1.0 & -0.8 & 0.0 & -0.2 \\
\hline$g(12,12)$ & -0.3 & 0.2 & -0.5 & -0.1 \\
\hline$h(12,12)$ & 0.7 & 0.9 & 0.8 & 0.4 \\
\hline$g(13,0)$ & 0.7 & 0.8 & 0.5 & -1.5 \\
\hline$g(13,1)$ & -1.1 & -1.5 & -1.3 & -1.2 \\
\hline$h(13,1)$ & -2.5 & -2.1 & -1.5 & -0.7 \\
\hline$g(13,2)$ & -1.5 & -0.7 & -0.7 & -0.1 \\
\hline$h(13,2)$ & -0.3 & -0.3 & 0.3 & 0.4 \\
\hline$g(13,3)$ & -0.1 & 0.1 & 0.1 & 0.4 \\
\hline$h(13,3)$ & 2.1 & 2.0 & 1.9 & 2.2 \\
\hline$g(13,4)$ & -0.5 & -0.4 & -0.4 & -0.3 \\
\hline$h(13,4)$ & -0.4 & -0.5 & -0.7 & -0.4 \\
\hline$g(13,5)$ & 1.4 & 1.2 & 1.3 & 1.4 \\
\hline$h(13,5)$ & -0.9 & -0.8 & -1.2 & -1.0 \\
\hline$g(13,6)$ & -0.4 & -0.4 & -0.6 & -0.4 \\
\hline$h(13,6)$ & -0.1 & 0.0 & -0.2 & 0.2 \\
\hline$g(13,7)$ & 0.5 & 0.5 & 0.5 & 0.6 \\
\hline$h(13,7)$ & 0.8 & 0.8 & 0.5 & 0.8 \\
\hline$g(13,8)$ & -0.3 & -0.2 & -0.4 & -0.5 \\
\hline$h(13,8)$ & 0.2 & 0.2 & 0.6 & 0.2 \\
\hline$g(13,9)$ & 0.2 & 0.3 & 0.2 & 0.1 \\
\hline$h(13,9)$ & 0.8 & 0.8 & 0.5 & 0.7 \\
\hline$g(13,10)$ & 0.0 & -0.1 & 0.1 & 0.0 \\
\hline$h(13,10)$ & 0.3 & 0.4 & 0.6 & 0.6 \\
\hline$g(13,11)$ & 0.7 & 0.5 & 0.2 & 0.4 \\
\hline$h(13,11)$ & -0.1 & 0.0 & -0.8 & -0.4 \\
\hline$g(13,12)$ & 0.2 & 0.2 & 0.4 & 0.2 \\
\hline$h(13,12)$ & -0.8 & -0.9 & -0.7 & -0.3 \\
\hline$g(13,13)$ & -0.3 & 0.0 & -0.2 & -0.1 \\
\hline$h(13,13)$ & -0.6 & -0.7 & -1.4 & -1.2 \\
\hline RMS Fit & 12.8 & 12.8 & 17.8 & 20.3 \\
\hline
\end{tabular}



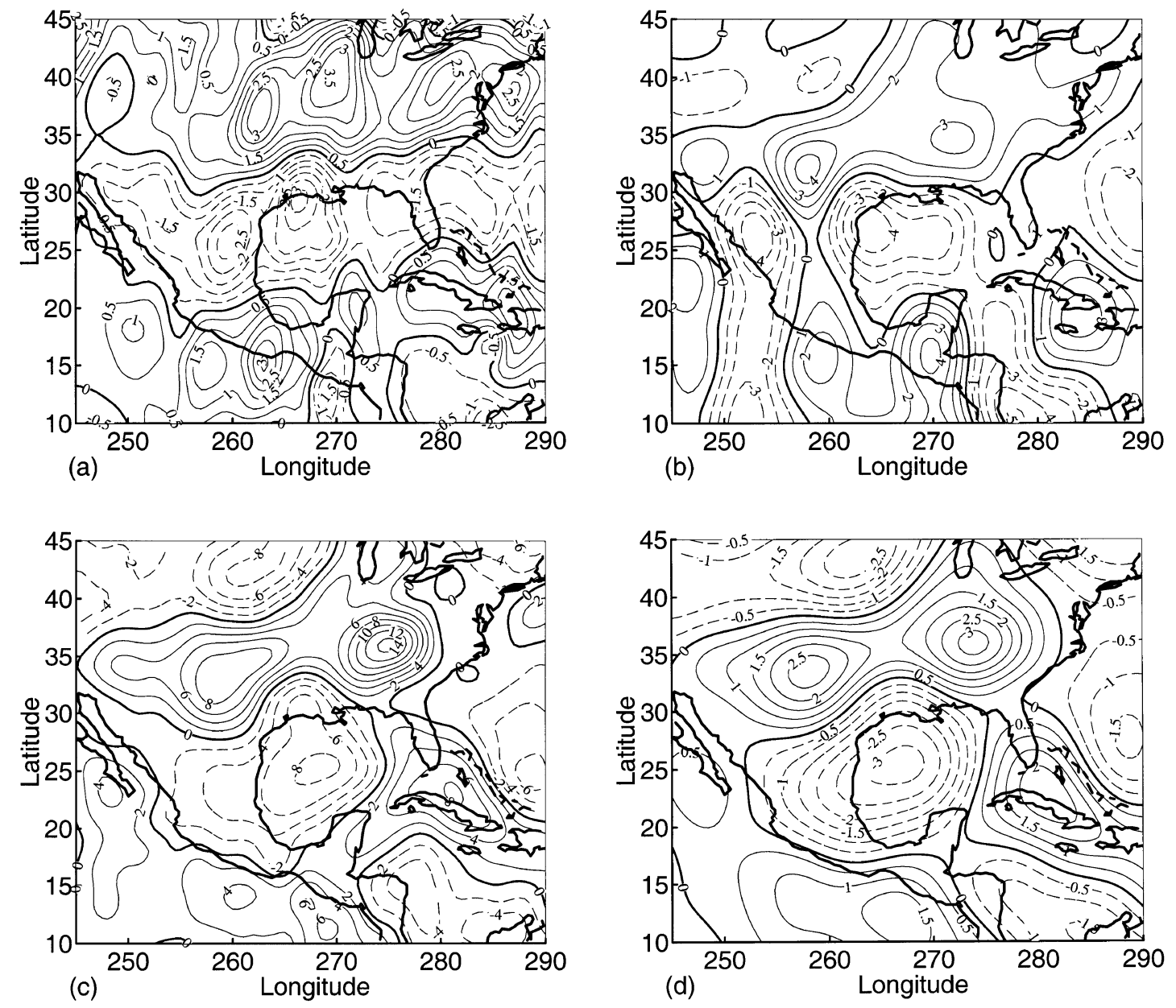

Fig. 13. (a) Ørsted scalar anomaly field at $7130 \mathrm{~km}$ when core field removed by along-track, high-pass filtering. (b) Ørsted scalar anomal field at $7130 \mathrm{~km}$ when core field removed by degree and order 13 model. (c) Magsat scalar anomaly at $6800 \mathrm{~km}$ from dawn data when core field removed by degree and order 13 model. (d) Magsat scalar anomaly field of Fig. 13(c) upward continued to $7130 \mathrm{~km}$.

filtering in Fig. 13(d).

\section{Results}

Table 1 gives the result of iterative spherical harmonic analysis of nighttime Ørsted OVH scalar data and MAG-L vector data for April, May, September, and December 1999, for a degree and order 13 internal core field and degree and order 1 external field. The external field is a uniform field with monthly variations of between 30 and $60 \mathrm{nT}$ directed southwards, parallel to the geomagnetic axis. It should be noted that only days with rms departures of observed values from the model values of less than $50 \mathrm{nT}$ were used in the calculations.

The Ørsted rms values noted for the months of April, May, September, and December are $12.8 \mathrm{nT}, 12.8 \mathrm{nT}, 17.8 \mathrm{nT}$ and $20.3 \mathrm{nT}$. These values are greater than the $8 \mathrm{nT}$ result given by Backus et al. (1996) for Magsat. The values could easily be reduced by choosing a value smaller than the 50 nT difference that has been allowed between core field and data. For monthly sections of Magsat data the rms values were $16 \mathrm{nT}$.

The month of September was more magnetically disturbed than April, May or December, giving a higher value for the external field term $\mathrm{G}(1,0)$ in September.

\section{Conclusions}

For latitudes below 50 degrees, the along-track high-pass filtered residuals produce regional scalar anomaly fields in better agreement with the corresponding plots produced from upward continued Magsat values than residuals obtained by subtraction of a degree and order 13 core field model computed from the available nighttime scalar and vector data. The along-track high-pass filtering of satellite observations gives scalar anomaly fields directly without the need for spherical harmonic analysis to determine the core magnetic field to degree and order 13.

Acknowledgments. Dr. Langel, Dr. Purucker, and the National Space Science Data Center (NSSDC) provided the Magsat data. Dr. Natalia Papitashvili and Dr. Joseph King have made the data available on CD_ROM with NSSDC ID of 79-094A-02V. The NSSDC web site is given below. Volume 9 of Geophysical Research Letters, 1982, has many papers devoted to preliminary analyses of Magsat data. We are grateful for the support of the Ørsted Project Office and the Ørsted Science Data Centre at the Danish Meteorological Institute. The Danish Ministry of Transport, Ministry of Research and Information Technology, and Ministry of Trade and Industry fund the Ørsted Project. Additional support was provided by National Aeronautics and Space Administration (NASA), European Space Agency (ESA), Centre Nationale d'Etudes Spatiales (CNES), and Deutsche Agentur fur Raumfahrtangelegenheiten (DARA). All 


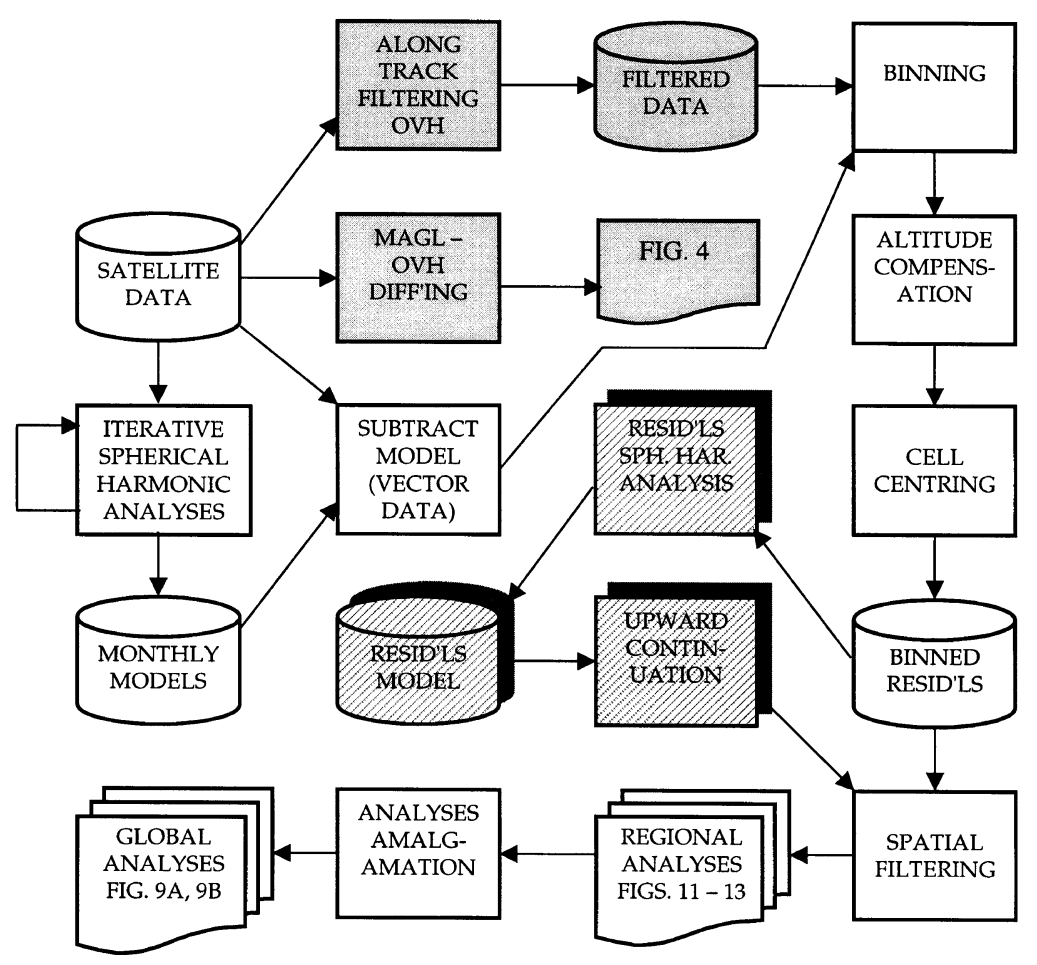

Fig. 14. Data processing flow chart of calculations. Plain boxes apply to both Ørsted and Magsat data. Grey filled boxes apply to Ørsted data only. Shaded boxes with light diagonal lines apply to Magsat data only. Cylinders represent data, squares represent calculations, and the triple files represent output of figures.

Ørsted information and data access is through the Ørsted website given below. Magsat data is also available through the Ørsted website. The Australian Research Committee Large Grant ARC A69702642 supported this research. The Australian Geological Survey Organization provided support for the completion of the analysis. We are indebted to the EPS Guest Editor, Dr. F. J. Lowes, and the referees, Dr. M. Purucker and Dr. C. Reeves for their comments and criticism.

Web sites

Magsat http://nssdc.gsfc.nasa.gov/space/; NASA NSSDC http://nssdc.gsfc.nasa.gov/cd-rom/; Ørsted http://web.dmi.dk/projects/oersted/

\section{References}

Arkani-Hamed, J., R. A. Langel, and M. Purucker, Magnetic anomaly maps of the Earth derived from POGO and Magsat data, J. Geophys. Res., 99, 24075-24090, 1994.

Backus, G., R. Parker, and C. Constable, Foundations of Geomagnetism, p. 140, Cambridge University Press, 1996.

Cain, J. C., Z. Wang, C. Kluth, and D. R. Schmitz, Derivation of a geomagnetic model to $n=63$, Geophys. J. Int., 97, 43-441, 1989.
Kaiser, J. F., Nonrecursive digital filter design using window function, in Proceedings of the 1974 IEEE International Symposium on Circuits and Systems, pp. 20-23, IEEE, New York, 1974.

Langel, R. A. and W. J. Hinze, The Magnetic Field of the Earth's Lithosphere, 429 pp., Cambridge University Press, 1998.

Olsen, N., Data for Ørsted Initial Field Model, e-mail to Ørsted main field investigators, 27 March 2000.

Olsen, N., L. Tffner-Clausen, R. Risbo, P. Brauser, J. Merayo, F. Primdahl, and T. Sabaka, In-flight calibration methods used for the Ørsted Mission, 2000.

Schuster, A., On some definite integrals and a new method of reducing a function of spherical co-ordinates to a series of spherical harmonics, Phil. Trans. R. Soc. Lond. A, 200, 181-223, 1903.

Wang, Z., Understanding models of the geomagnetic field by Fourier analysis, J. Geomag. Geoelectr., 39, 333-347, 1987.

Winch, D. E., Analysis of the geomagnetic field by means of Cartesian components, Phys. Earth Planet. Int., 1, 347-360, 1968.

D. J. Ivers (e-mail: ivers_d@maths.usyd.edu.au), R. J. Stening, J. Turner, and D. E. Winch 